\title{
Gender difference in the coronary risk factors amongst the patients with Acute Coronary Events (ACE) in Nepal
}

\author{
B. Rawat, Abhinav Baidya, Jesson Gurung, Subhash Acharya, Minalma \\ Pandey, Pragya Verma, Pooja Poudyal, H.S. Sodhi, D. B. Karki, S. C. \\ Jha and Dr. Rajib Pande
}

\section{Introduction:}

The onset of symptomatic CAD is typically about 10 years earlier in men, but CAD incidence in women increases rapidly at menopause. Women have the same modifiable risk factors as that of men. Although Diabetes appears to confer greater risk factors as that of men as may low HDL, cholesterol and plasma triglyceride. Not much data is available to asses If coronary risk factors are different in male Vs female population in South Asia.

\section{Method and observation:}

A prospective analysis was done in 518 consecutive patients admitted with Acute coronary Events (ACE) in CCU in our institute from January 1999 to November 2003. The data was analyzed and various risk factors were satisfied.

Out of 518 patients of ACE, 388 (65.25) patients were male and 180 (34.74\%) patients were female. The commonest risk factors amongst male patients was smoking in 209 (61.83), hypertension in 197 (58.28\%) dyslipidemia in, 156 (46.15\%) followed by diabetes in $109(32.24 \%)$ and a positive family history in $84(24.85 \%)$, Against this the female patients had commonest risk factors, hypertension in 96 (53.33), diabetes in 81 (45\%) and dyslipidemia in $33(18.33 \%)$. 
- NEPALESE HEART JOURNAL

\section{Conclusion:}

Women suffering from Acute Coronary Event are more likely to be older and to have the history of hypertension and diabetes and are less likely to be smokers than their male counterparts. 